\title{
Studies on Antimicrobial and Antioxidant Potential of Metal Complexes and Synthetic Organic Compounds
}

\author{
Saira Zahoor* and Muhammad Sajid Tanoli \\ Department of Biochemistry, Hazara University Mansehra, Pakistan \\ *Corresponding Author: Saira Zahoor, Department of Biochemistry, Hazara University Mansehra, Pakistan.
}

Received: May 23, 2019; Published: September 23, 2019

DOI: 10.31080/ASMS.2019.03.0412

\begin{abstract}
Organic compounds and metal complexes play important roles in biological system, and it has been recognized that many synthetic organic compounds and metal complexes used in manufacturing of medicines and are bio transformed by metal ions metabolism. Studies on metal compounds of Schiff bases and synthetic compounds have been of major significance due to their spectral properties and vast applications. The current study deals with the compounds and determination of their antibacterial and antioxidant activities and on the basis of results, three compounds at various concentrations give indicatively high scavenging capacity. In scavenging capacity, results of the three compounds which are metal complexes having codes are SZ-4-9, SZ-4-11 and SZ-4-13 at different concentrations (10 ppm, 100 ppm, 1000 ppm) were statistically significant. Similarly, results of lower DPPH scavenging capacity were also significant in compounds having code KH-4-3. Out of twenty-three only four compound having codes SZ-4-3, SZ-4-4, SZ-4-5 and SZ-4-9 showed the encouraging results against four bacterial strains.
\end{abstract}

Keywords: Metal Complexes; Synthetic Organic Compounds; Antibacterial Activity Antioxidant Activity; Ascorbic Acid; Ceftriaxone Sodium

\section{Introduction}

Metal complexes are groups of atoms having chemical structure in which medial metal atom is encircle by metalloid atoms called ligands which are joined by chemical bonding [1]. Metals play avital role in the manufacturing of different drugs for years, ever since human being started to walk on the planet. Many metals are very essential for our body which our diets contain in varying quantities, although people have only recently realized importance of metals. It happened due to increased in knowledge education and awareness of people, personnel and family health. Metal complexes are used in current medical use in many classes of the pharmaceuticals [9]. Some metal complexes having strong antimicrobial activities which are already running in the market [2,3]. The compounds which do not occur naturally and prepared by the reaction of other compound are known as synthetic compounds. Herbal medicines are mostly used for the traditional treatment of health problems in developing countries [4]. New organic synthetic compounds synthesized in laboratory and used in the manufacturing of drugs [5]. Bacteria consist of a large domain of prokaryotic microorganisms. Bacteria consist of multiple shapes, like spheres to rods and spirals, a few micrometers in length. Bacteria were among the first life forms to appear on Earth. Its habitat is soil, water, acidic hot springs, radioactive waste [8] and the deep portions of Earth's crust. Escherichia coli are known as facultative anaerobic gramnegative bacteria [10]. It is rod like in structure which is normally found in the warm-blooded organisms [7]. Staphylococcus aureus is a gram-positive coccal bacterium. It is a member of the Firmicutes. Klebsiella pneumoniae is gram-negative, nonmotile, encapsulated, rod-shaped bacterium. They grow in clusters and have no flagella [12]. Streptococcus pneumoniae is a gram - positive bacteria. Metal complexes are the sunny reddish color which was firstly introduced in India and famous to old Egyptians [14]. Schiff base complexes and synthetic organic compounds have taken more attention recently in bioinorganic and biochemistry due to their antimicrobial and chemotherapy features [13]. To some extent metals having showed the antitumor efficacy like zinc and cadmium [15-17]. 


\section{Materials and Methods}

To investigate the antioxidant and antibacterial activity of the synthetic organic compounds and metal complexes were studied by methods discuss below.

\section{Materials}

The following chemicals and compounds were used.

Collection of synthetic organic compounds, metal complexes and chemicals

Samples of metal complexes and synthetic organic compounds were collected from the chemistry lab of Hazara University Mansehra. These compounds were already identified by using Fourier Transform Infrared (FT-IR) and Nuclear Magnetic Resonance (NMR) spectroscopies and their molecular weights were measured by Mass Spectrometry (MS). The compounds are listed below in table 1.

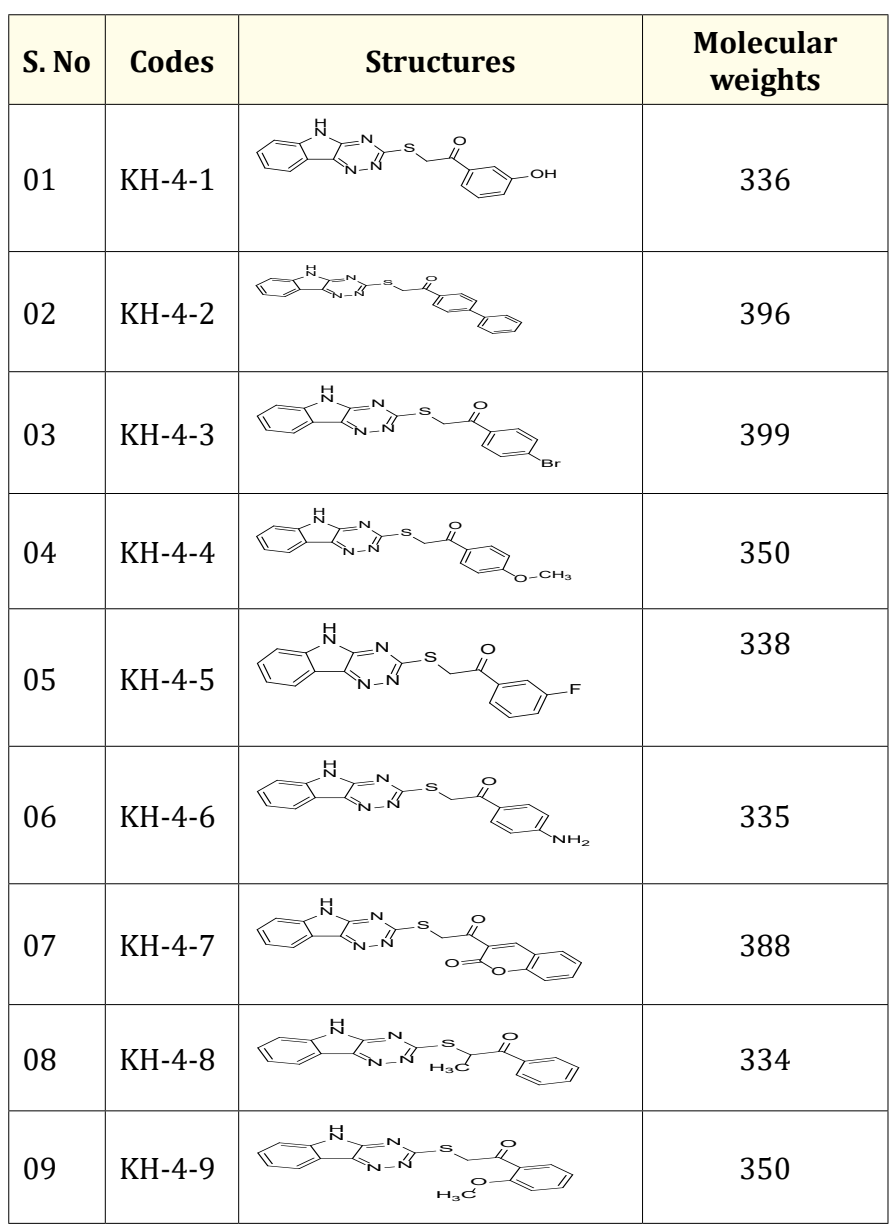

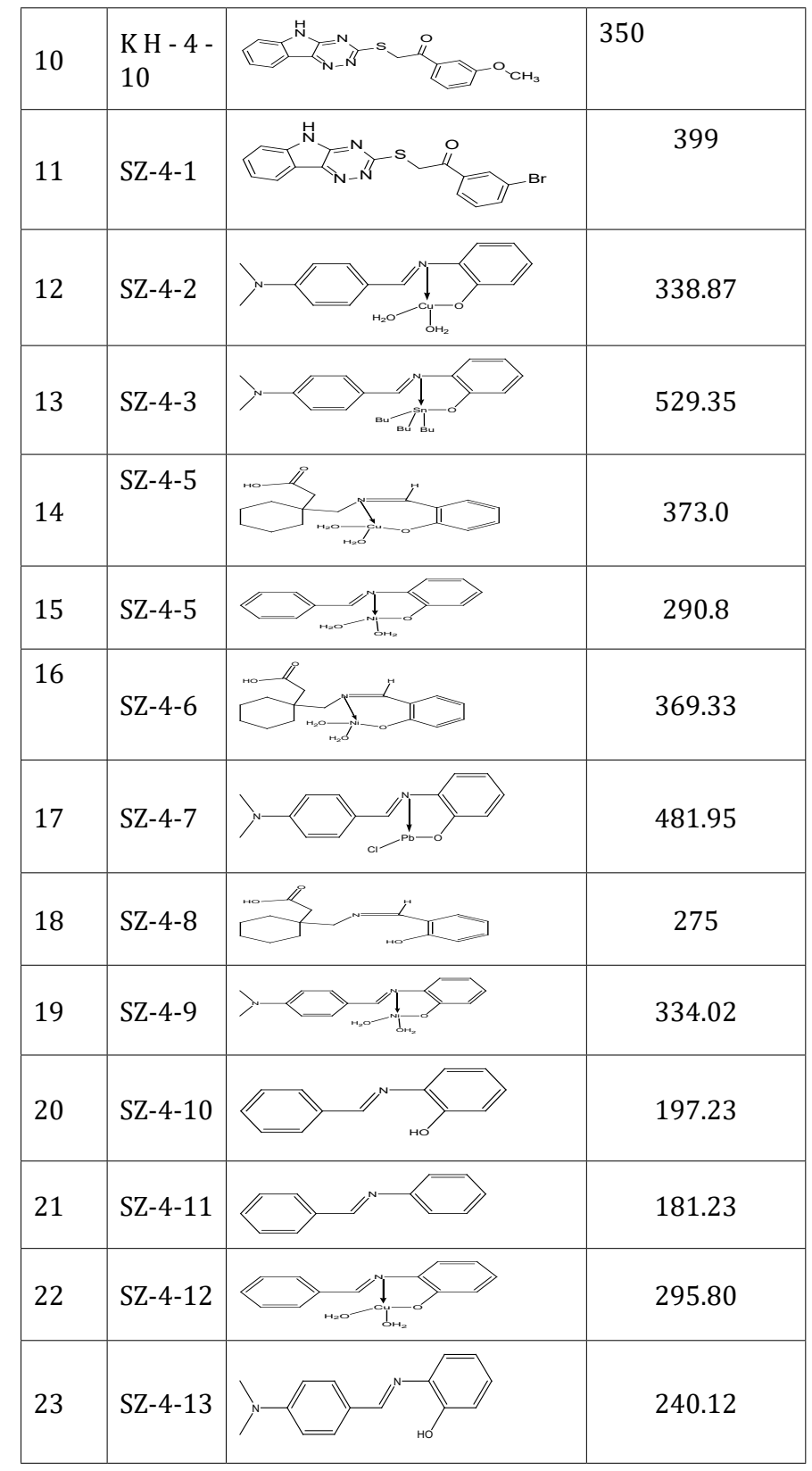

Table 1: Synthetic organic compounds and metal complexes.

\section{Materials}

2, 2-diphenyl-1-picrylhydrazyl (DPPH) (Sigma), Methanol (HPLC Grade), Ethanol (HPLC Grade) Ascorbic acid $\left(\mathrm{C}_{6} \mathrm{H}_{8} \mathrm{O}_{6}\right.$ ) (Sigma), Test samples (Chemistry lab of Hazara University Mansehra) UV spectrophotometer (UV/VIS China) and Micropipette (Accumax). Nutrient agar (Oxoid), Culture of bacterial strains (Biola- 
bs Islamabad), Test samples (Chemistry lab of Hazara University Mansehra), Hot Incubator (Forma scientific), Cold Incubator (Forma scientific), Laminar Floor Hood (Galvano Scientific), Sodium Hydroxide (Sigma), Autoclave (Galvano Scientific), Hot plate magnetic stirrer (DHPS-1) Dry heat sterilizer (DHG9030A), Weighing balance (AUX220 shimadzu), Disinfectant Isopropyl Alcohol (I.P.A 70\%) (Sigma), Micropipette (Accumax).

\section{Microorganisms used}

Four strains of bacteria were selected on the bases of their clinical importance which were already identified, and out of which two were gram negative i.e. Klebsiella pneumoniae and E. coli and two bacteria were gram positive Streptococcus pneumoniae and Staphylococcus aureus. These microorganisms were collected from Bio labs Islamabad were inoculated on nutrient agar media at $4^{\circ} \mathrm{C}$ temperature. The stock cultures were maintained at $4^{\circ} \mathrm{C}$.

\section{Methodology}

Antioxidant and antibacterial activity of metal complexes and synthetic organic compounds were studied by the following methods.

\section{Antioxidant activity}

The antioxidant activities of all synthetic compounds and metal complexes were determined by DPPH scavenging activity.

\section{DPPH scavenging activity}

The method used to detect the antioxidant activity of all synthetic organic compounds and metal complexes was described by [6] modified by [11].

\section{Preparation of stock solution}

Bulk solution of DPPH was prepared by dissolving $32 \mathrm{mg}$ DPPH reagent in $1000 \mathrm{ml}$ of $80 \%$ methanol. Bulk solution of ascorbic acid (positive control) was prepared in water by dissolving $0.15 \mathrm{mg} /$ $\mathrm{mL}, 1.5 \mathrm{mg} / \mathrm{mL}$, and $15 \mathrm{mg} / \mathrm{mL}$ in ethanol for $150 \mathrm{ppm}, 1500 \mathrm{ppm}$ and $15000 \mathrm{ppm}$ respectively. The solution of all samples was prepared with strength of $0.15 \mathrm{mg} / \mathrm{mL}, 1.5 \mathrm{mg} / \mathrm{mL}$, and $15 \mathrm{mg} / \mathrm{mL}$ in ethanol for $150 \mathrm{ppm}, 1500 \mathrm{ppm}$ and $15000 \mathrm{ppm}$.

\section{Procedure}

After preparation of stock solution $200 \mu \mathrm{L}$ of the test sample and $3 \mathrm{~mL}$ of DPPH solution were taken in test tube for each sample from stock solution. The final concentration of samples became 10ppm, 100 ppm, 1000 ppm by using formula M1V1=M2V2. Mixtures were shacked gently and kept in dark for one hour at room temperature. After one-hour samples were run on UV spectrophotometer along with standard ascorbic acid simutansiously by using $80 \%$ methanol was as a blank. Absorbance for all test samples and positive control i.e ascorbic acid were measured at $517 \mathrm{~nm}$ by using UV spectrophotometer. DPPH was used as a negative control whereas ascorbic acid was used as a positive control and methanol $80 \%$ was used as a blank. Test was performed in triplicate for each sample.

The final percentage inhibition was calculated by the following formula.

DPPH scavenging activity (Percentage inhibition $)=[$ Ac - As $/ \mathrm{Ac}]$ $\times 100$

$\mathrm{Ac}=$ Absorbance of control

As $=$ Absorbance of sample

The results were averages of triplicate for each sample.

\section{Antibacterial activity}

In this study antibacterial activity of metal complexes and synthetic organic compounds were observed by using well diffusion method against four different pathogenic bacterial strains i.e. Staphylococcus aureus, Streptococcus pneumoniae, E. coli, and Klebsiella pneumoniae. Staphylococcus aureus and Streptococcus pneumoniae were gram positive bacteria and E. coli, and Klebsiella pneumoniae were gram negative bacteria.

\section{Standard drug dilutions}

Ceftriaxone sodium salt was used as positive control by dissolving $62 \mathrm{mg}$ standard drug of ceftriaxone in $625 \mu \mathrm{L}$ of ethanol that the concentration becomes $62 \mathrm{mg} / 625 \mu \mathrm{L}$ for $1: 10$ and 62 $\mathrm{mg} / 1225 \mathrm{~mL}$ for 1:20 dilutions, and ethanol were used as negative control.

Sample preparation of synthetic organic compounds and metal complexes

Bulk solution of different compound was prepared by dissolving $62 \mathrm{mg} / 625 \mu \mathrm{L}$ ethanol for 1:10 dilution and $62 \mathrm{mg} / 1250 \mathrm{~mL}$ of ethanol for 1:20 dilutions. 
Studies on Antimicrobial and Antioxidant Potential of Metal Complexes and Synthetic Organic Compounds

Sub culturing of bacterial strains and pour samples

Weared gloves and mask and mopped the hands with methanol. Mopped the laminar flow hood with disinfectant methanol. Took out the petri dishes from incubator and placed on the bench of laminar flow hood. Took petri dish and bacterial culture was seeded on media with the help of sterile swab stick. Well was made in the center of petri plates with the help of micropipette tip. Then filled whole well fill with $50 \mu \mathrm{L}$ sample of test dilution, ethanol was used as a negative control. Plates were kept in hybridization oven at $37^{\circ} \mathrm{C}$ for 24 hours. Clear zones of inhibition of test samples were measured by using Vernier caliper after 24 hours. For each sample duplicate plates were prepared having two different dilutions (1:10 and 1:20). Ethanol was used as a negative control for against four bacterial strains (Klebsiella pneumoniae, E. coli, Streptococcus pneumoniae and Staphylococcus aureus).

\section{Results and Discussion}

Bacteria can cause different diseases in human, animals and plants. Several drugs are used against these bacteria due to heavy reliance on these drugs several strains developed resistance so there is need to search and develop new antibacterial agents. In present study we investigated the antimicrobial activity of synthetic compounds. In addition to this we evaluated the antioxidant activity of these compounds.

\section{Antibacterial activity}

The antimicrobial potency of the synthetic organic compounds and metal complexes were studied in different concentrations (62 $\mathrm{mg} / 625 \mathrm{~mL}$ and $62 \mathrm{mg} / 1250 \mathrm{~mL}$ ) for 1:10 and 1:20 dilutions respectively by well diffusion method against four pathogenic bacterial strains. Two were Gram-positive Staphylococcus aureus ATCC No 2592 and Streptococcus pneumoniae ATCC No 49619, and two were Gram-negative Escherichia coli ATCC No 25922 and Klebsiella pneumoniae ATCC No 10031. Based on their applications these bacterial strains have been selected and the purpose of further formulation study. Antibacterial activity of metal complexes and synthetic organic compounds were assessed in the form of zone of inhibition of bacterial growth. The results are shown in the following table 2 for 1:10 and 1:20 dilutions. Antibacterial activities of synthetic organic compounds and metal complexes were checked against four bacterial strains Staphylococcus aureus, Streptococcus pneumoniae, Escherichia coli and Klebsiella pneumoniae. The com- pounds having codes $\mathrm{KH}-4-1, \mathrm{KH}-4-2, \mathrm{KH}-4-3, \mathrm{KH}-4-4, \mathrm{KH}-4-5, \mathrm{KH}-$ 4-6, KH-4-9, KH-4-10 and SZ-4-10 did not show any activity against above four bacterial strains on 1:10 and 1:20 dilutions. Compounds having code SZ-4-4, SZ-4-5, SZ-4-7 and SZ-4-9 were active against four bacterial strains Staphylococcus aureus, Streptococcus pneumoniae, Escherichia coli and Klebsiella pneumoniae and showed the encouraging results. In comparison of gram positive and gramnegative bacterial strains the code SZ-4-2 and SZ-4-3 showed much sensitivity to gram negative bacterial strain i.e. Klebsiella pneumoni$a e$ and showed encouraging zones of inhibitions on 1:10 and 1:20 dilutions. SZ-4-12 showed the best antibacterial activity against Klebsiella pneumoniae and showed $12.6 \mathrm{~mm}$ and $12 \mathrm{~mm}$ zones of inhibition respectively. It did not show good activity against rest bacterial strains Escherichia coli, Staphylococcus aureus and Streptococcus pneumoniae. SZ-4-3 showed $7 \mathrm{~mm}$ and $5.8 \mathrm{~mm}$ zone of inhibition against Staphylococcus aureus $7 \mathrm{~mm}$ and $6 \mathrm{~mm}$ zone of inhibition against Streptococcus pneumoniae. In case of gramnegative bacteria sample SZ-4-12 showed the best antibacterial activity and gave $12.6 \mathrm{~mm}$ and $12 \mathrm{~mm}$ zones of inhibition against Klebsiella pneumoniae. In case of KH-4 series KH-4-7 showed the highest zone of inhibition against Streptococcus pneumoniae which is $3.3 \mathrm{~mm}$, but it was much less as compare to standard drug ceftriaxone. In case of metal complexes, the sample having code SZ4-3 and SZ-4-12 showed highest zones of inhibition among all the compounds against Klebsiella pneumoniae but rest were not much sensitive to this strain of bacteria. When we compare overall results then out of twenty-three only four compound having codes SZ-4-3, SZ-4-4, SZ-4-5 and SZ-4-9 showed the encouraging results against four bacterial strains. Ceftriaxone is a new third generation cephalosporin with excellent activity against many gram-negative and gram-positive microorganisms was used as standard drug. The values obtained are the mean of three independent replicate along with standard deviation because the activity was performed in triplicate. Results are shown in table 2 for 1:10 and 1:20 dilutions. In antibacterial activity when we compare the samples with standard the sample having code SZ-4-3 and SZ-4-12 showed the encouraging results and both having $12.6 \mathrm{~mm}$ and $12 \mathrm{~mm}$ zone of inhibition on Klebsiella pneumoniae on 1:10 and 1:20 dilutions. The both samples showed the best activity on 1:10 and 1:20 dilution as compared to standard drug ceftriaxone sodium which having 12.5 $\mathrm{mm}$ and $11 \mathrm{~mm}$ zone of inhibition on 1:10 and 1:20 dilutions. 


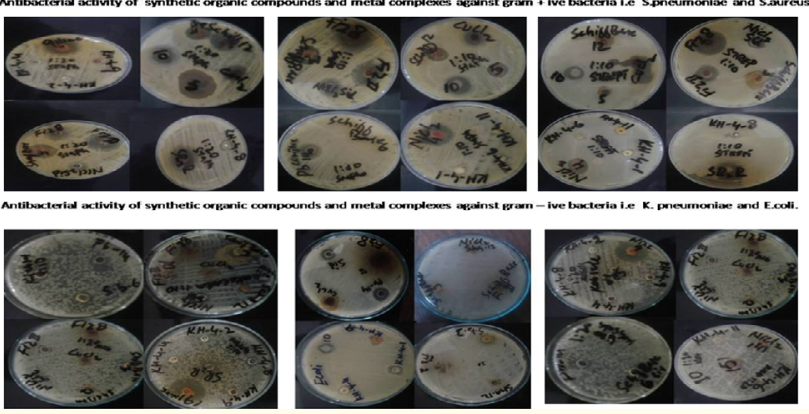

Figure 1: Antibacterial activity of synthetic organic compounds and metal complexes against four pathogenic strains.

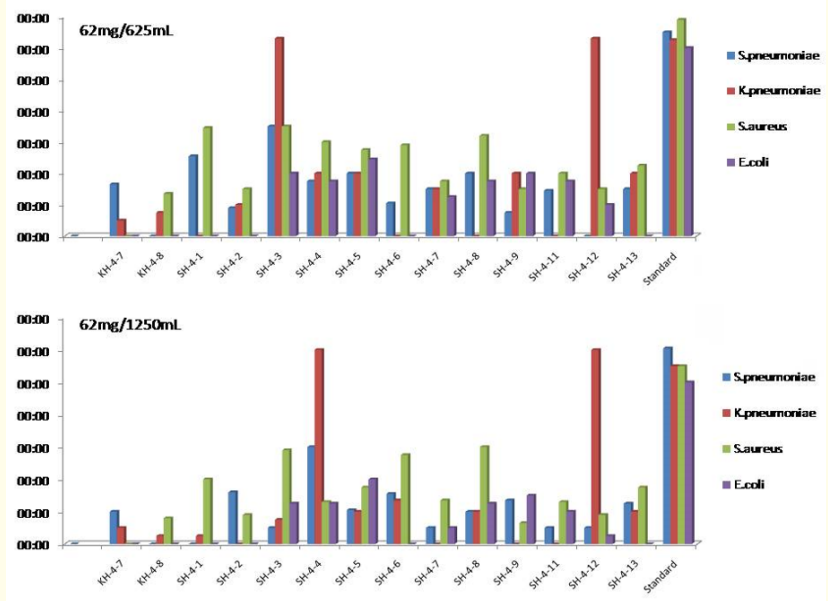

Figure 2: Antibacterial activity of synthetic organic compounds and metal complexes.

\section{Antioxidant activity}

Antioxidant activity of all compounds and metal complexes were checked in three different dilutions. All dilutions of synthetic organic compounds of $\mathrm{KH}-4$ series showed decrease in DPPH percentage scavenging capacities as compared to their standard which is mentioned as positive control i.e. Ascorbic acid except KH-4-7 which showed highest scavenging capacities as compared to other compounds in $10 \mathrm{ppm}, 100 \mathrm{ppm}$, and $1000 \mathrm{ppm}$ dilutions. In case of metal complexes three samples having codes SZ-4-11, SZ-4-13 and SZ-4-9 showed increase in DPPH percentage scaveng- ing capacities as compared to their standard $10 \mathrm{ppm}, 100 \mathrm{ppm}$ and 1000 ppm dilutions.

We have performed antioxidant activity in twenty-three samples with different dilutions. SZ-4-13 showed the highest antioxidant capacity which was $99 \%$ among all the compounds comparative to ascorbic acid which was reference standard which showed 98.1\% potency. When we compared DPPH scavenging activities with standard ascorbic acid then we observed other compounds also showed satisfactory results which can be seen in table 2 . In case of KH-4 series only KH-4-7 showed the best results and gave $75.3 \%$ potency which was considerable but less than from ascorbic acid and in SZ series SZ-4-1, SZ-4-6, SZ-4-9, SZ-4-11 showed the encouraging results among all the dilution. When we compare overall results the metal complexes showed the best results as compare to synthetic organic compounds are shown in figure 3.

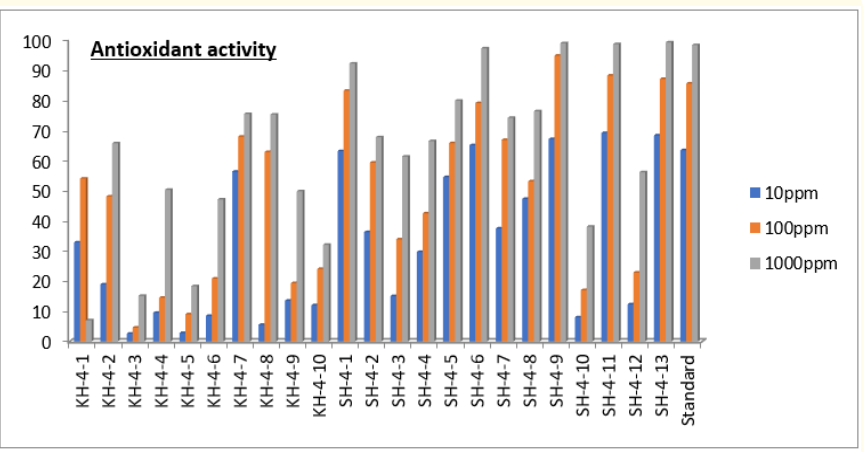

Figure 3: DPPH scavenging activity of synthetic organic compounds and metal complexes at $10 \mathrm{ppm}, 100 \mathrm{ppm}$ and 1000 ppm dilutions.

\section{Conclusion and Future Strategies}

The presence of important synthetic organic compounds as well as metal complexes suggested that these compounds possess varying degree of biological activities including antibacterial and antioxidants activities and can be further studied for the structure illumination and development of pharmacologically important medicinal chemistry of agents.

Metal complexes and synthetic organic compounds showed the best activity against different bacterial strains and can be used as 


\begin{tabular}{|c|c|c|c|c|}
\hline \multirow{4}{*}{ S.No } & \multirow{4}{*}{ Samples code } & \multicolumn{3}{|c|}{ DPPH Percentage scavenging activity } \\
\hline & & \multicolumn{3}{|c|}{ Percentage Inhibition } \\
\hline & & \multicolumn{3}{|c|}{ (10 ppm,100 ppm,1000 ppm) $0.00015 \mathrm{~g} / \mathrm{mL}, 0.0015 \mathrm{~g} / \mathrm{mL}, 0.0015 \mathrm{~g} / \mathrm{mL} \pm \mathrm{S}$. Dev } \\
\hline & & 10ppm & 100ppm & 1000ppm \\
\hline 1 & KH-4-1 & $32.8 \pm 0$ & $53.9 \pm 0.0015$ & $7 \pm 0$ \\
\hline 2 & KH-4-2 & $18.9 \pm 0$ & $48 \pm 0.0015$ & $65.6 \pm 0.0005$ \\
\hline 3 & $\mathrm{KH}-4-3$ & $2.59 \pm 0.005$ & $4.59 \pm 0.001$ & $15.1 \pm 0.0005$ \\
\hline 4 & $\mathrm{KH}-4-4$ & $9.5 \pm 0.005$ & $14.5 \pm 0$ & $50.2 \pm 0.001$ \\
\hline 5 & $\mathrm{KH}-4-5$ & $2.8 \pm 0.0005$ & $9 \pm 0.0015$ & $18.3 \pm 0$ \\
\hline 6 & $\mathrm{KH}-4-6$ & $8.49 \pm 0.001$ & $20.8 \pm 0.001$ & $47 \pm 0$ \\
\hline 7 & KH-4-7 & $56.2 \pm 0.0005$ & $67.8 \pm 0.005$ & $75.3 \pm 0.001$ \\
\hline 8 & $\mathrm{KH}-4-8$ & $52.5 \pm 0.001$ & $62.7 \pm 0.001$ & $75.1 \pm 0.0005$ \\
\hline 9 & $\mathrm{KH}-4-9$ & $13.5 \pm 0.005$ & $19.31 \pm 0.005$ & $49.7 \pm 0$ \\
\hline 10 & KH-4-11 & $12 \pm 0.001$ & $24 \pm 0.005$ & $32 \pm 0.0005$ \\
\hline 11 & SZ-4-1 & $63 \pm 0.005$ & $83 \pm 0.0005$ & $92 \pm 0.001$ \\
\hline 12 & SZ-4-2 & $36.2 \pm 0$ & $59.2 \pm 0.005$ & $67.6 \pm 0.001$ \\
\hline 13 & $S Z=4-3$ & $15 \pm 0.001$ & $33.8 \pm 0.0005$ & $61.2 \pm 0.0005$ \\
\hline 14 & SZ-4-4 & $29.64 \pm 0.001$ & $42.4 \pm 0.001$ & $66.3 \pm 0$ \\
\hline 15 & SZ-4-5 & $54.4 \pm 0.005$ & $65.64 \pm 0.0005$ & $79.7 \pm 0$ \\
\hline 16 & SZ-4-6 & $64.98 \pm 0.005$ & $78.9 \pm 0.005$ & $97 \pm 0.0005$ \\
\hline 17 & SZ-4-7 & $37.4 \pm 0.001$ & $66.7 \pm 0.005$ & $74 \pm 0.0005$ \\
\hline 18 & SZ-4-8 & $47.2 \pm 0$ & $53 \pm 0.001$ & $76.2 \pm 0.005$ \\
\hline 19 & SZ-4-9 & $67 \pm 0$ & $94.6 \pm 0.51$ & $98.7 \pm 0.051$ \\
\hline 20 & SZ-4-10 & $8 \pm 0.001$ & $17 \pm 0.001$ & $38 \pm 0.005$ \\
\hline 21 & SZ-4-11 & $69 \pm 0$ & $88 \pm 0$ & $98.4 \pm 0.0005$ \\
\hline 22 & SZ-4-12 & $12.3 \pm 0.005$ & $22.8 \pm 0.001$ & $56 \pm 0$ \\
\hline 23 & SZ-4-13 & $68.2 \pm 0$ & $86.9 \pm 0.005$ & $99 \pm 0.0005$ \\
\hline 24 & Ascorbic acid & $66.3 \pm 0.005$ & $85.4 \pm 0.0005$ & $98.1 \pm 0$ \\
\hline
\end{tabular}

Table 2: Antioxidant activity of synthetic organic compounds and metal complexes.

antibacterial agents to inhibit the bacterial growth or to kill the pathogenic microorganisms against different infectious diseases. Some metal complexes and synthetic organic compounds showed the best antioxidant activity can also be used as antioxidants to removal of oxygen.

\section{Bibliography}

1. Akter S., et al. "Solvent effects on antioxidant properties of persimmon (Diospyros kaki L. cv. Daebong) seeds". International Journal of Food Science and Technology 45 (2010): 2258-2264.
2. Barry AL. "The antimicrobic susceptibility test". Principles and Practices (1976): 95-99.

3. Lea and Febiger. Antimicrobial Agents and Chemotherapy. Copyright $(\mathcal{C}$ American Society for Microbiology Printed in U.S.A 13.1 (1978): 61-69.

4. Bedir E., et al. "Biologically active steroidal glycosides from Tribulus terrestris". Pharmazie 57.1 (2002): 491-493. 
5. Casewell M and Talsania HG. "Predominance of certain klebsiella capsular types in hospitals in the United Kingdom". Journal of Infection 1 (1979): 77-79.

6. Kulisic T., et al. "Use of different methods for testing antioxidative activity of oregano essential oil". Food Chemistry 85 (2004): 633-640.

7. Latif A and Shaker R. "The chemistry of mercapto- and thionesubstituted 1, 2, 4-triazoles and their utility in heterocyclic synthesis". Journal of Heterocyclic Chemistry 26 (1989): 769.

8. Li H and Sun H. "Recent advances in bioinorganic chemistry of bismuth". Current Opinion in Chemical Biology 16.1-2 (2012): 74-83.

9. Lippert B and Cisplatin. "Chemistry and Biochemistry of a Leading Anticancer Drug". John Wiley and Sons: Amsterdam, The Netherlands, Molecules 20 (2015): 7955.

10. Mishra, N., et al. "An overview of biological aspects of Schiff base metal complexes". International Journal of Advancements in Research and Technology 2.8 (2013): 52-66.

11. Obeid HK., et al. "Bioactivity and analysis of biophenols recovered from olive mill waste". Journal of Agricultural and Food Chemistry 53.4 (2005): 823-837.

12. Peru and Bolivia. "Escherichia coli in children". Journal of Emerging Infectious Diseases 12.6 (2006): 375-379.

13. Pizarro AM and Sadler PJ. "Unusual DNA binding modes for metal anticancer complexes". Biochimie 91.10 (2009): 11981211.

14. Raman, N., et al. "Synthesis and spectral characterization of antifungal sensitive Schiff base transition metal complexes". Mycobiology 35.3 (2007): 150-153.

15. Rohaya A., et al. "Antioxidant, radical-scavenging, anti-inflammatory, cytotoxic and antibacterial activities of methanolic extracts of some Hedyotis species". Life Sciences 76.17 (2005): 1953-1964.
16. Rosenberg B., et al. "Platinum compounds: A new class of potent antitumour agents". Nature 222 (1969): 385-386.

17. Ryan KJ and Ray CG. "Sherris Medical Microbiology". McGraw Hill (2005).

\section{Volume 3 Issue 10 October 2019}

(C) All rights are reserved by Saira Zahoor and Muhammad Sajid Tanoli . 